\title{
P I5-09. The potential global market size and public health value of hypothetical HIV-I preventive vaccines with different levels of efficacy
} CA Marzetta*1, SJ Wrobel ${ }^{1}$, KK Singh², ND Russell ${ }^{2}$ and J Esparza ${ }^{2}$

Address: ${ }^{1}$ Applied Strategies Consulting, San Mateo, CA, USA and ${ }^{2}$ The Bill \& Melinda Gates Foundation, Seattle, WA, USA

* Corresponding author

from AIDS Vaccine 2009

Paris, France. 19-22 October 2009

Published: 22 October 2009

Retrovirology 2009, 6(Supp| 3):P210 doi:10.1 I86/I742-4690-6-S3-P210

This abstract is available from: http://www.retrovirology.com/content/6/S3/P2I0

(c) 2009 Marzetta et al; licensee BioMed Central Ltd.

\section{Background}

This study evaluated the potential global market size and value of a hypothetical preventive HIV-1 vaccine that takes into account clade specificity, partial prevention of infection, impact on transmission, delay to treatment, and healthcare system differences regarding HIV-1 screening and its impact on potential market size.

\section{Methods}

The potential market size and value of a hypothetical HIV1 vaccine effective against $H I V-1$ clades $A, B$ and $C$ was assessed across five different potential product profiles using an Excel-based spreadsheet model. Vaccine efficacy (prevention of acquisition and/or decrease in virus load), disease burden, clade distribution, vaccine price, market segment, vaccine delivery infrastructure, and HIV screening capabilities were taken into account. It was assumed that high disease burden countries (HDBC) where HIV prevalence rates are $>1 \%$ would routinely vaccinate adolescents, whereas low disease burden countries (LDBC) with HIV prevalence rates $<1 \%$ would routinely vaccinate high-risk groups only. Routine vaccination and one-time catch-up campaigns were assessed.

\section{Results}

Potential demand and market value was seen across all product profiles and increased with disease impact (e.g., decrease on HIV-1 transmission and delay in time to treatment) and increased efficacy $(0 \%-60 \%)$. The overall potential demand during the routine and catch-up cam- paign vaccination period was estimated to be $130-270$ million peak annual doses in HDBC and 20-60 million peak annual doses in LDBC. The potential value was related to the product profile but was greater than $\$ 200$ million annually, even for a vaccine expected to modestly decrease transmission and delay treatment by 1-2 years.

\section{Conclusion}

This study evaluated in detail the value of a multi-clade HIV-1 vaccine taking into account vaccine, disease, and healthcare complexities. The findings of this study provide donors and suppliers new data to consider in their continuing efforts to develop an HIV-1 vaccine that addresses clades relevant to worldwide disease burden. 